\title{
Experiências Maternas no Contexto da Prematuridade: um Estudo de Revisão Sistemática
}

Experiencias Maternas en el Contexto de la Prematuridad: un Estudio de Revisión Sistemática Maternal Experiences in the Context of Prematurity: a Systematic Review Study

Denice Bortolin

ORCID: https://orcid.org/0000-0003-1891-1075 Universidade do Vale do Rio dos Sinos, Rio Grande do Sul/Brasil

Tagma Marina Schneider Donelli

ORCID: https://orcid.org/0000-0003-3083-0083

Universidade do Vale do Rio dos Sinos, Rio Grande do Sul/Brasil

Carine Tabaczinski

ORCID: https://orcid.org/0000-0002-9622-752X

Faculdade Meridional, Rio Grande do Sul/Brasil

Declaração de Direito Autoral

A submissão de originais para este periódico implica na transferência, pelos autores, dos direitos de publicação impressa e digital. Os direitos autorais para os artigos publicados são do autor, com direitos do periódico sobre a primeira publicação. Os autores somente poderão utilizar os mesmos resultados em outras publicações indicando claramente este periódico como o meio da publicação original. Em virtude de sermos um periódico de acesso aberto, permite-se o uso gratuito dos artigos em aplicações educacionais e científicas desde que citada a fonte conforme a licença CC-BY da Creative Commons.

Creative Commons Atribuição 4.0 Internacional.

\section{Resumo}

O parto do recém-nascidos pré-termo (RNPT) ocorre quando há o nascimento de um bebê antes das 37 semanas de gestação. O RNPT tem maior propensão a apresentar problemas clínicos, o que faz com que tenha de ficar internado na Unidade de Terapia Intensiva Neonatal (UTIN). Tal situação acaba impactando no desenvolvimento do vínculo mãe-bebê e, consequentemente, nas experiências maternas. Objetivou-se realizar uma revisão sistemática da literatura, mapeando os estudos empíricos sobre as experiências maternas em situação de prematuridade. Utilizou-se como termos de busca "premature or preterm birth and maternal experiences", apenas na versão em inglês. Considerou-se os artigos empíricos disponibilizados integralmente e publicados em inglês ou português, advindos da psicologia, nos últimos cinco anos (janeiro de 2013 a janeiro de 2018). A busca se realizou em janeiro e fevereiro de 2018. Os resultados foram analisados através de dez categorias: tipos de publicação; ano de publicação; continente e país de origem do artigo; formação acadêmica dos pesquisadores; idioma predominante nas publicações; objetivos do estudo; delineamento; participantes; instrumentos; e resultados. Foram encontrados seis artigos, em sua maioria publicados em revistas multidisciplinares, por autores da área da medicina, com delineamento qualitativo, na língua inglesa, tendo como foco principal a avaliação dos serviços prestados pela equipe de saúde na UTIN. Houve somente um artigo escrito por psicólogos, evidenciando-se, assim, a necessidade de inserção maior deste profissional neste contexto.

Palavras-chave: Prematuridade; Experiências maternas; Revisão sistemática. 


\title{
Resumen
}

El parto prematuro se produce cuando el nacimiento de un bebé antes de las 37 semanas de gestación. El bebé prematuro tiene mayor propensión a presentar problemas clínicos, lo que hace que tenga que quedarse internado en la Unidad de Terapia Intensiva Neonatal (UTIN), y tal situación acaba impactando en el desarrollo del vínculo madre-bebé y, consecuentemente, en las experiencias maternas. La meta és realizar una revisión sistemática de la literatura, que mapean los estudios empíricos sobre las experiencias maternas en situación de prematuridad. Se utilizó como términos de búsqueda "premature o preterm birth y maternal experiences". Se consideró los artículos empíricos totalmente disponibles y publicados en Inglés o portugués, que viene de la psicología en los últimos cinco años (enero 2013-enero 2018). La búsqueda se realizó en enero y febrero de 2018. Los resultados analizaron diez categorías: tipos de publicación; año de publicación; continente y país de origen del artículo; formación académica de los investigadores; el idioma predominante en las publicaciones; objetivos del estudio; diseño; los participantes; instrumentos; y resultados. Se encontraron seis artículos, en su mayoría publicados en revistas multidisciplinares, por autores del área de la medicina, con delineamiento cualitativo, teniendo foco principal, la evaluación de los servicios prestados por el equipo de salud en la UTIN. Sólo hubo un artículo escrito por psicólogos, evidenciándose, así, la necesidad de inserción mayor de este profesional en este contexto.

Palabras clave: Prematuridad; Experiencias maternas; Revisión sistemática.

\begin{abstract}
Preterm labor occurs when a baby is born before 37 weeks of gestation. The preterm labor more likely to present clinical problems, which means that he or she must be admitted to the neonatal intensive care unit (NICU), and this situation has an impact on the development of the mother-infant bond and consequently on maternal experiences. The objective was to carry out a systematic review of the literature, mapping the empirical studies about maternal experiences in a situation of prematurity. It was used as search terms "premature or preterm birth and maternal experiences", only in the English version. We considered the empirical articles made available in full and published in English or Portuguese, coming from psychology, in the last five years. The search was carried out in January and February of 2018. The results were analyzed through ten categories: types of publication; year of publication; mainland and country of origin of the article; academic training of researchers; predominant language in publications; study objectives; design; participants; instruments; and results. Six articles were found, mostly published in multidisciplinary journals, by authors of the medical field, with a qualitative design, in English, with the main focus being the evaluation of the services provided by the health team in the NICU. There was only one article written by psychologists, evidencing, therefore, the need for greater insertion of this professional in this context.
\end{abstract}

Keywords: Prematurity; Maternal experiences; Systematic review.

\section{Introdução}

A prematuridade, que é definida como o nascimento antes das 37 semanas de gestação, é a principal causa de mortalidade em crianças menores de cinco anos de idade em todo o mundo (Word Health Organization [WHO], 2018). O Ministério da Saúde (2011a) assinala a prematuridade como a principal causa de mortalidade infantil no primeiro mês de vida de crianças brasileiras, sendo que $70 \%$ destas ocorrem nos primeiros 28 dias. O Brasil é o $10^{\circ}$ país em número de nascidos vivos prematuros, e o $16^{\circ}$ em óbitos por complicações da prematuridade. De outra parte, ressalta-se que a prevalência de $11,7 \%$ de partos prematuros em relação aos partos realizados no Brasil torna a prematuridade um importante problema de saúde pública no país (Ministério da Saúde, 2011a; Unicef Brasil, 2013). 
Uma pesquisa constatou que as mães de recém-nascidos pré-termo (RNPT) apresentam uma maior fragilidade psíquica, pelo fato de estarem antecipando a maternidade, sem vivenciar todo o período da gestação (Fernandes, Lamy, Morsch, Lamy Filho, \& Coelho, 2011). Quando estes bebês permanecem em UTIN, as percepções maternas podem ser influenciadas negativamente por estereótipos de prematuridade, e os bebês passam a ser vistos como pouco maduros, fisicamente menos potentes, menos sociáveis e com menos capacidades cognitivas (GonzálezSerrano et al., 2012).

Um estudo de revisão sistemática sobre os fatores de risco da prematuridade, realizado por Almeida, Lins, Camelo e Mello (2013), aponta que as pessoas com nascimento prétermo estão sujeitas a problemas futuros, pela sua condição de precocidade. Esses problemas surgem em consequência da imaturidade de órgãos, e de danos causados pelo baixo peso ao nascer. O RNPT pode desenvolver problemas momentâneo ao nascimento, como hiperbilirrubinemia ${ }^{1}$, e também problemas na vida adulta, como, por exemplo, risco aumentado para doenças cardiovasculares.

A pesquisa de Almeida et al. (2013) descreve, ainda, que uma parte expressiva dos artigos encontrados foram publicados em 2010, demonstrando que a prematuridade tem sido um tema de interesse recente, e talvez por este motivo se justifique a escassez de estudos na área. De outra parte, a contemporaneidade do assunto pode estar associada ao aumento da sobrevida de crianças nascidas pré-termo, possibilitada pelo avanço tecnológico na área da saúde nos últimos anos (Almeida, Lins, Camelo e Mello, 2013).

Entretanto, percebe-se uma carência de dados sobre os efeitos psicológicos causados pela condição de prematuridade, dados estes que servem para o planejamento de estratégias de intervenção de caráter preventivo e curativo.
Diante dessa lacuna, torna-se fundamental discutir a prematuridade, e, mais especificamente, as experiências maternas em relação à prematuridade de um filho, já que as primeiras relações entre a mãe e o bebê poderão influenciar a formação ou fortalecimento dos laços afetivos, sendo estas relações iniciais o protótipo das relações futuras do indivíduo (Bowlby, 1997).

Nos casos de prematuridade, existe para a mãe um forte sentimento de ameaça de morte, de dedicar-se a um bebê que pode não sobreviver, que não pode ser intitulado como seu. Sobre isso, Wanderley (1997) comenta que os pais de RNPT vivenciam o encontro inicial atravessados por aparelhos e monitores, entre medicamentos e exames, e estes influenciam diretamente a relação mãe-bebê. Neste contexto hospitalar, instala-se uma "preocupação médica primária", em contraposição ao termo Winnicotiano de "preocupação materna primária", assim, esta mãe fica impactada por este discurso hospitalar, apresentando dificuldades em criar seu discurso sobre o bebê, e passa a significá-lo de acordo com os termos técnicos do profissional da área médica (Wanderley, 1997). Para Ferrari e Donelli (2010), esse discurso pode impossibilitar as primeiras identificações que a mãe deve ter com seu filho para realizar os seus desejos e necessidades, pois o significante que permeia esse bebê é de um ser frágil, que pode vir a falecer, e que, por isso, exige uma série de cuidados especiais, com o propósito de amenizar as implicações do parto do RNPT

Além disso, nos nascimentos a termo, a mãe tem de realizar um movimento de emparelhamento entre o bebê da sua imaginação e o bebê real, que acaba de nascer. Esse é um processo natural e comum em todas as mães, pois o bebê imaginário nunca é como o bebê real, e, por isso, a puérpera passa por um processo de luto (Lebovici, 1987; Soulé, 1987). No entanto, nos casos de nascimento pré-termo,

\footnotetext{
1 Hiperbilirrubinemia neonatal: doença que surge no bebê logo após o nascimento, causada pelo acúmulo de bilirrubina no sangue, deixando a pele do bebê amarelada (Moreira, Lopes, \& Carvalho, 2004).
} 
essa tarefa se torna ainda mais complexa aos novos pais, pois o contato real com o bebê pode provocar um significativo impacto, pois, na maioria das vezes, o recém-nascido é pequeno, pálido, fraco, frágil e imaturo, bem diferente do bebê imaginário (Brazelton \& Cramer, 1992). Ainda, de acordo com Stern (1997), o filho, no momento do nascimento, cria uma neoformação psíquica nos pais, indicando que a inclusão do bebê no psiquismo parental determina transformações intensas e irreversíveis.

Diante desse complexo universo da maternidade na prematuridade, o presente artigo objetivou realizar uma revisão sistemática da literatura, nos meses de janeiro e fevereiro de 2018, mapeando os estudos empíricos sobre as experiências maternas em situação de prematuridade publicados entre janeiro de 2013 e janeiro de 2018.

\section{Método}

Esta revisão sistemática da literatura foi desenvolvida de acordo com as diretrizes do Preferred Reporting Itens for Systematic Reviews and Meta-Analyses (Moher, Liberati, Tetzlaff, Altman, The PRISMA Group, 2009), que avalia as características de redação do estudo e não necessariamente sua qualidade metodológica, sendo composto por um conjunto de 27 itens sobre informações que devem ser claramente descritas no artigo. $\mathrm{O}$ protocolo do PRISMA pode ser acessado pelo endereço eletrônico http://www.prismastatement.org/. A identificação e seleção dos artigos em todas as bases de dados foram realizadas simultaneamente por dois pesquisadores durante o mês de janeiro e fevereiro de 2018.

\section{Estratégia de Busca}

As bases e portais de publicações eletrônicas selecionados para o estudo foram Lilacs, BVS, Scielo, Psycinfo, Scopus, Medline, Pepsic e Capes, pois estas bases publicam, preferencialmente, estudos na área da saúde, foco do presente estudo, e foram utilizados os descritores padronizados em inglês: "premature OR preterm birth" AND "maternal experiences". Foi utilizada somente a expressão em inglês por se considerar a língua inglesa como uma língua universal, bem como pelo fato de que a grande maioria dos artigos publicados, independentemente da sua língua, apresentam o abstract em inglês. Utilizou-se, ainda, a expressão booleana "AND", a fim de localizar os registros com ocorrência simultânea entre os descritores referidos, e a palavra "OR" para realizar uma busca pelos termos "pré-termo" ou "nascimento prematuro", o que possibilitou a obtenção de um maior alcance na temática.

\section{Seleção dos Estudos}

Visando uma seleção mais eficiente de artigos, foram utilizados alguns critérios de inclusão, considerando-se: (a) ser artigo empírico, científico e relacionado à pesquisa; (b) ter sido publicado no período de janeiro de 2013 a janeiro de 2018 (últimos cinco anos); (c) o material estar disponibilizado virtual, integral e gratuitamente; e (d) estar disponibilizado em inglês e/ou português.

Como critérios de exclusão, optou-se por excluir: (a) itens que estavam publicados em formato de tese, dissertação, livro, capítulo de livro, resenha, artigo teórico, relato de experiência, estudo de caso e revisão sistemática ou de literatura; (b) itens que não estavam relacionados à temática "experiências maternas de bebês prematuros"; (c) itens cuja experiência materna ocorresse com bebês a termo; (d) estudos de experiências de mães de RNPT nos contextos de saúde geral, que fogem do alcance da psicologia; (e) artigos não disponíveis em inglês ou português; e (f) artigos sem texto completo ou resumo disponível.

Para fins de seleção, após a aplicação dos critérios de inclusão e exclusão, foram descartadas as publicações em duplicidade, por meio da leitura dos títulos das mesmas. Para a elegibilidade, os textos foram selecionados pelo título e resumo, e, posteriormente, os eleitos foram lidos na íntegra. 


\section{Processo de Extração de Dados}

Os estudos que contemplaram os critérios de inclusão foram analisados de forma descritiva e qualitativa. Com o objetivo de realizar uma análise aprofundada da produção científica encontrada, estabeleceram-se categorias que demarcaram o exame do material, como: 1) "tipos de publicação", considerando a natureza e a frequência de cada tipo; 2) "ano de publicação"; 3 ) "continente e pais de origem"; 4)" formação acadêmica dos pesquisadores" 5) "idioma de publicação"; 6) "objetivos do estudo"; 7) "delineamento"; 8) "participantes"; 9) "instrumentos; e 10) "resultados"; Assim, seguiu-se uma análise quantitativa das categorias, buscando identificar a frequência de cada item, e qualitativa, visando apreciar o conteúdo das mesmas. Dentre estas categorias, foram analisadas as convergências e divergências entre as publicações, selecionando os temas mais recorrentes.

\section{Risco de Viés entre os Estudos}

A identificação e a seleção dos artigos nas bases de dados foram realizadas por dois pesquisadores, de forma independente e sistemática, os quais fizeram a identificação inicial pelos títulos das publicações encontradas pelos descritores e, posteriormente, pelos resumos obtidos por busca eletrônica. Após essa seleção, cada pesquisador realizou a leitura na íntegra dos artigos selecionados, e, posteriormente, elencou os artigos possíveis de compor a revisão. Uma nova avaliação foi realizada pelos dois pesquisadores, que determinaram, de forma consensual, os estudos a serem incluídos na revisão.

$\mathrm{Na}$ Figura 1, é apresentado o procedimento realizado para seleção dos itens de análise.

Primeiramente, a busca nas bases de dados de estudos publicados em inglês e português, efetuada durante os meses de janeiro e fevereiro de 2018, resultou no total de 11.217 artigos, todos referentes aos últimos 05 anos (janeiro de 2013 a janeiro de 2018). Após essa investigação inicial, relacionou-se os artigos a partir dos critérios de inclusão e exclusão, previamente estabelecidos. Conforme fluxograma:

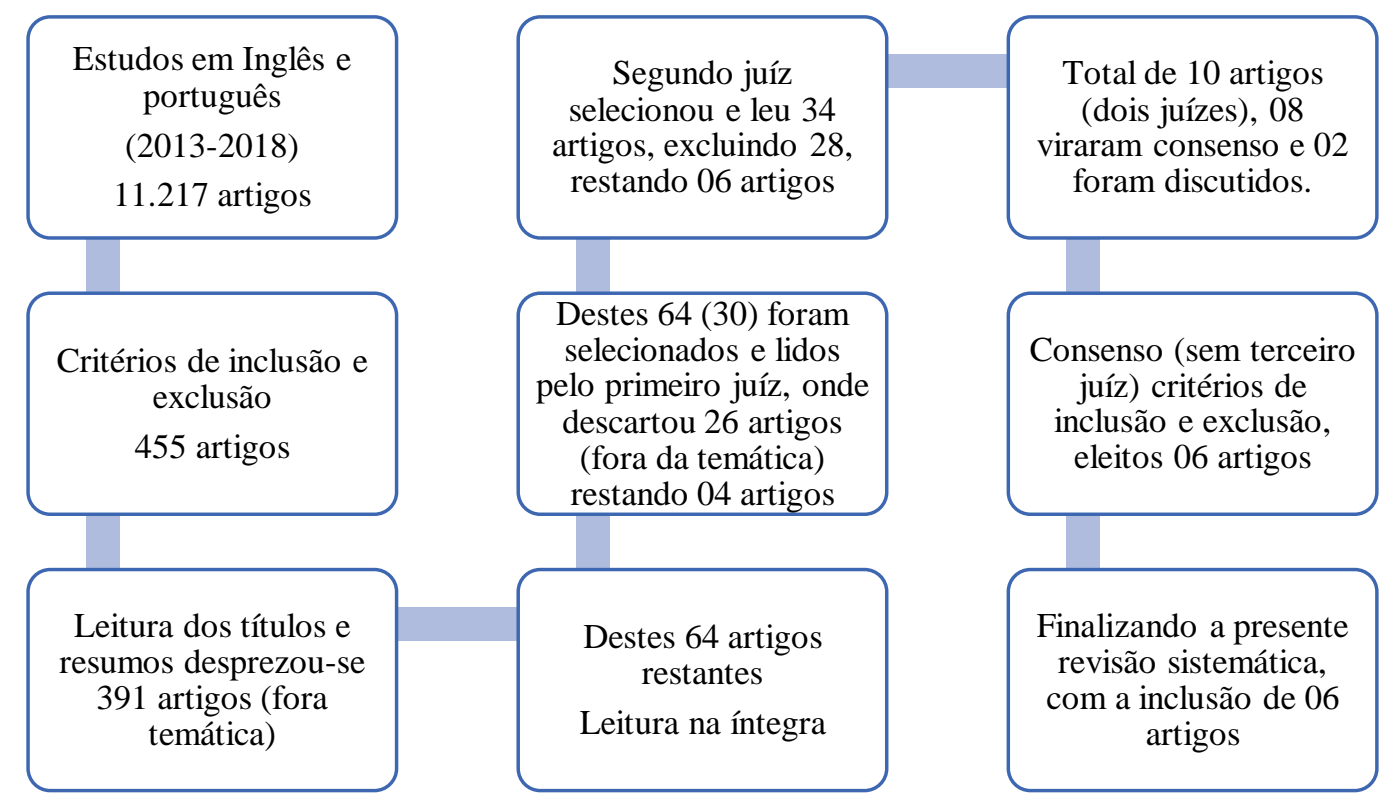




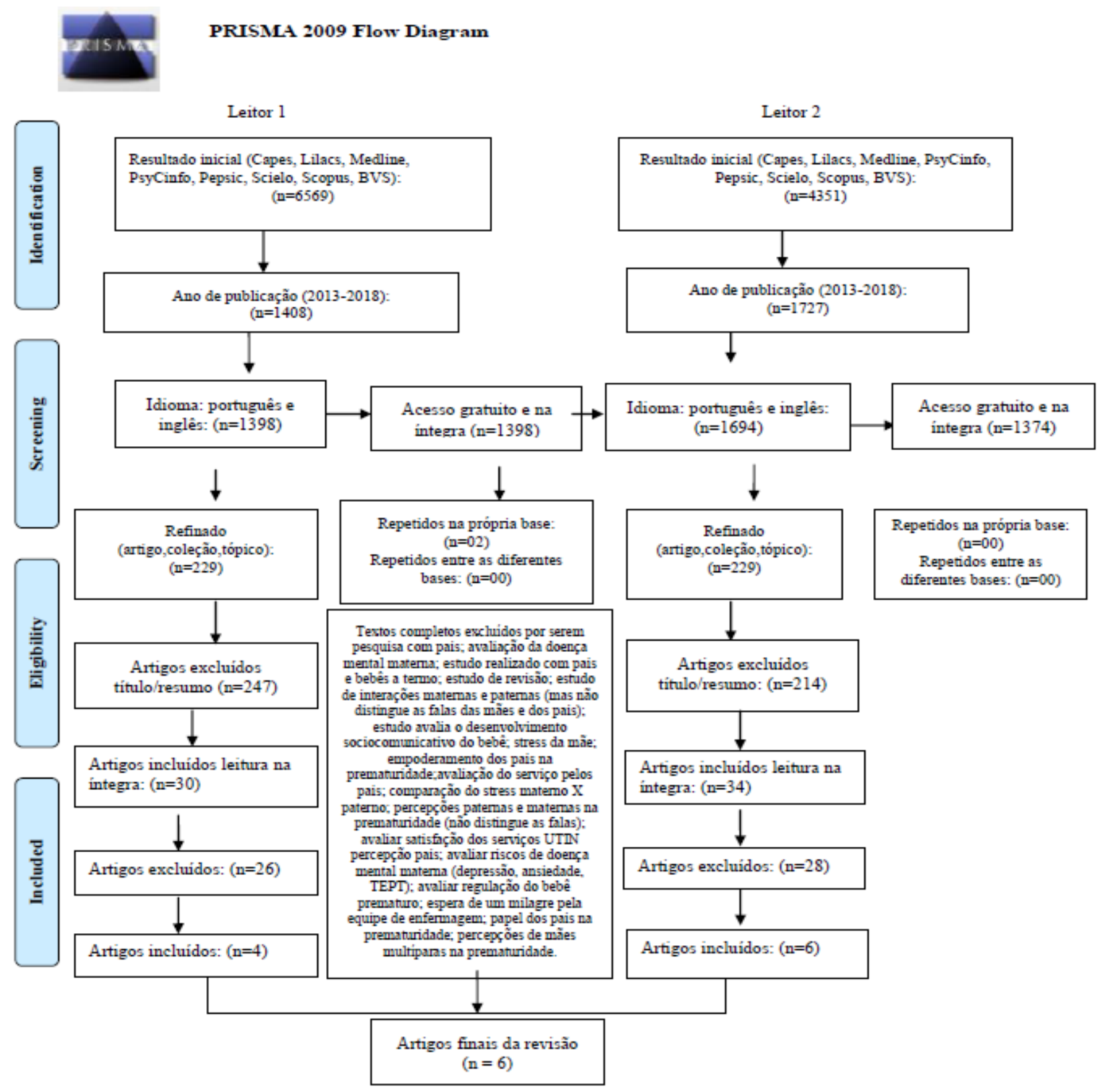

Figura 1. Fluxograma da seleção de artigos para a revisão sistemática

Nota. Fonte: Adaptado de "Principais itens para relatar Revisões sistemáticas e Meta-análises: A recomendação PRISMA" de T.F. Galvão., T de S. A. Pansani e D. Harrad, 2015, Epistemologia e Saúde, 24(2). doi: 10.5123/S167949742015000200017

\section{Resultados e Discussão}

Buscando uma análise aprofundada da produção científica pesquisada, estabeleceramse categorias que nortearam o exame do material, como: 1) "tipo de publicação", considerando a natureza e a frequência de cada tipo; 2) "ano de publicação"; 3 ) "continente e país de origem"; 4) "formação acadêmica dos pesquisadores"; 5) "idioma de publicação"; 6) “objetivos do estudo"; 7) "delineamento"; 8) "participantes"; 9) "instrumentos"; e 10) "resultados". Para tal aprofundamento, seguiuse uma análise quantitativa das categorias, com o objetivo de identificar a frequência de cada item, e uma qualitativa, buscando contemplar o conteúdo das mesmas.

A respeito da categoria 1, "tipo de publicação", sua natureza e frequência, 
encontrou-se um artigo publicado na Revista de Saúde Sexual e Reprodutiva de Homens e Mulheres, um artigo publicado na Revista de Enfermagem Pediátrica, dois artigos publicados na Revista de Pediatria, outros dois em revistas multidisciplinares em ciências naturais, pesquisa médica, engenharia, ciências sociais, tecnologia, humanidades e artes. Os dados demonstram que a maioria dos artigos (05) foram publicados em revistas da área médica, e somente um artigo foi publicado na área da enfermagem, evidenciando que há uma preferência dos pesquisadores em publicarem seus estudos na área da medicina.

A categoria 2, "ano de publicação", deu origem a uma subcategoria denominada de "frequência de publicações por ano" (2.1). Nesta, verificou-se que o ano 2016 foi o que apresentou mais publicações, com três artigos selecionados ao todo (Hagen, Iversen, \& Svindseth, 2016; Nelson \& Bedford, 2016; Spinelli et al., 2016). Nos demais anos (2013, 2014 e 2017) houve o mesmo número de publicações do citado ano (Finlayson, Dixon, Smith, Dykes, \& Flacking, 2014; Guillaume et al., 2013; Premji et al., 2017), sendo uma em cada ano, não tendo ocorrido nenhuma publicação no ano de 2018. Diante disso, infere-se que o tema gerou maior interesse no ano de 2016. No entanto, a presente pesquisa realizou a revisão no início de 2018, não permitindo conhecer o real dado de publicações no decorrer deste ano.

Em relação à categoria 3, "continente e país de origem do artigo", destacou-se pesquisadores do continente Europeu (Inglaterra, Noruega, Itália e França), com cinco estudos divulgados entre 2013 a 2016; tendo sido encontrado somente um artigo publicado no continente americano (Canadá), em 2017. Apesar do Brasil ter o dobro da taxa de nascimentos prematuros em relação à Europa, vê-se que, no continente europeu, principalmente na Inglaterra (com publicação de dois artigos, 2014 e 2016), há um maior número de publicação acerca experiências maternas em contexto de prematuridade. Já com relação ao continente americano, onde houve a divulgação de somente um estudo, em virtude de se tratar de uma publicação recente (2017), talvez possa se pensar, que, naquele país, pode estar havendo um maior interesse pelo tema nesse período, apesar do mesmo constar na lista dos países com maior número de nascimentos prematuros (http://eportuguese.blogspot.com.br/2013/11/d ia-mundial-da prematuridade.html).

Quanto à categoria 4, “formação acadêmica dos pesquisadores", duas pesquisas foram realizadas por médicos (Guillaume et al., 2013; Hagen et al., 2016); um estudo foi realizado por enfermeiros (Nelson \& Bedford, 2016); outro estudo foi realizado por psicólogos (Spinelli et al., 2016); e duas pesquisas foram realizadas por uma equipe multidisciplinar (nutricionista, médico, educador e enfermeiro) (Finlayson et al., 2014; Premji et al., 2017). Evidencia-se que a equipe médica se destaca em termos de publicação, com dois artigos publicados. Contudo, esse número pode ser considerado maior, pois as equipes multidisciplinares, que eram formadas também por médicos, publicaram dois estudos, o que revela que o interesse da equipe médica por este tema é maior, neste período de realização do estudo. Desse modo, pode-se considerar que houve, de fato, quatro publicações com a participação de profissionais da medicina, sugere-se, de acordo com as pesquisas citadas no presente artigo, principalmente nos estudos (Finlayson et al., 2014; Nelson \& Bedford, 2016; Premji et al., 2017), que esses profissionais estão evidenciando a importância dos aspectos emocionais e relacionais no cuidado da saúde do RNPT

Sobre a categoria 5, "idioma predominante", a língua inglesa prevaleceu em todas as pesquisas desta revisão, tendo ocorrido nos seis artigos encontrados (Finlayson et al., 2014; Guillaume et al., 2013; Hagen et al., 2016; Nelson \& Bedford, 2016; Premji et al., 2017; Spinelli et al., 2016). Este resultado pode ter ocorrido, pois os descritores de busca da pesquisa, foram todos utilizados no idioma inglês, possivelmente esta escolha por parte da pesquisadora, pode ter influenciado os resultados. 
A categoria 6, "objetivo dos estudos", verificou que os diferentes estudos contemplaram objetivos muito semelhantes, e que vem ao encontro da temática do presente estudo, podendo ser analisado em três subcategorias: 6a) Estudos que privilegiaram a análise das experiências maternas (Finlayson et al., 2014; Premji et al., 2017; Spinelli et al., 2016), totalizando três estudos nesta perspectiva; 6b) Artigos que abordaram a percepção das experiências dos pais por meio da avaliação do serviço de saúde (Guillaume et al., 2013; Hagen et al., 2016), totalizando dois artigos; e 6c) Estudos que investigaram para além das experiências dos pais, preocupando-se com a compreensão de como a equipe de saúde está capacitada para o cuidado nos casos de prematuridade (Guillaume et al., 2013; Nelson \& Bedford, 2016), totalizando dois estudos. Percebe-se, então, que os estudos têm se preocupado com a consequência da prematuridade na tríade mãe-pai-bebê, e essa preocupação é extremamente pertinente, pois a constituição da vida psíquica do bebê se dá por meio desta relação (Winnicott, 2006). Além disso, destacam a fundamental importância que a equipe de profissionais de saúde tem no cuidado de mães, pais e bebês, visto que, se a equipe for uma "mãe" suficientemente boa para a mãe, esta também poderá ser uma mãe suficientemente boa para o bebê.

$\mathrm{Na}$ análise da categoria 7, "delineamento dos estudos", os dados revelaram que cinco estudos utilizaram o método qualitativo de pesquisa, e apenas um utilizou o método misto. Este significativo dado revela que os seis estudos foram qualitativos, pois, para compreensão das experiências maternas no contexto da prematuridade, esse parece ser o método mais eficiente para se obter os dados e alcançar o objetivo da pesquisa. Uma pesquisa qualitativa é definida por buscar a compreensão mais aprofundada da realidade, que não pode ser mensurada através de variáveis numéricas. Assim, ela busca explicações na dinâmica das relações, e o seu universo de trabalho são os sentimentos, crenças, valores, significados e motivos (Silveira \& Córdova, 2009).
$\mathrm{Na}$ análise da categoria 8, "participantes", gerou-se três subcategorias: 8a) "díade mãe-bebê"; 8b) "tríade mãe-paibebê" e 8c) "equipe de cuidados". $\mathrm{Na}$ subcategoria 8a), destacaram-se três artigos (Finlayson et al., 2014; Premji et al., 2017; Spinelli et al., 2016). Na subcategoria 8b), evidenciaram-se duas pesquisas, indicando que, ainda que os estudos demonstrem mais interesse em pesquisar a díade, há uma ocorrência de estudos interessados em pesquisar o papel dos pais no cuidado e na atenção à mãe e ao bebê, quando internado em UTINs. Isto pode estar relacionado ao pósparto, pois, logo após a ocorrência do mesmo, a mãe, em razão da sua condição de saúde, fica impedida de estar em contato direto com o bebê. Ressalta-se que alguns estudos têm revelado que a participação dos pais nos cuidados de UTIN tem auxiliado muito as mães a sentirem-se mais seguras e menos estressadas no cuidado com seus bebês. Inclusive, um destes estudos foi encontrado na presente revisão (Hagen et al., 2016). E, na categoria $8 \mathrm{c}$ ), equipes de cuidado, foram contemplados dois estudos, apontando para a preocupação em avaliar os serviços prestados aos pais e ao RNPT, e também o impacto que estes profissionais da saúde têm no bom desenvolvimento e na alta do bebê, bem como no desenvolvimento emocional da sua família. Os estudos revelam que muitos profissionais não estão aptos a trabalhar neste contexto, mesmo que alguns hospitais ofereçam treinamento para taL. Nestes casos, muitos profissionais não aderem ao protocolo do treinamento, constatação divulgada no estudo de Guillaume et al. (2013). A falta de preparação técnica e disponibilidade emocional dos profissionais da área de saúde é preocupante, uma vez que estes desempenham um papel fundamental no vínculo mãe-bebê em contexto de prematuridade, e, consequentemente, podem influenciar negativamente as experiências maternas, como retratam os estudos de Finlayson et al. (2014), Guillaume et al. (2013), Nelson e Bedford (2016), Premji et al. (2017) e Spinelli et al. (2016). 
$\mathrm{Na}$ categoria 9, "instrumentos", o que prevaleceu, na totalidade das pesquisas, ou seja, nos seis estudos, foi o uso de entrevistas semiestruturadas. Juntamente com estas, foram utilizadas fichas de dados sociodemográficos e prontuários médicos. No estudo de Nelson e Bedford (2016), que investigou o significado da maternidade de um bebê pré-termo que recebe cuidados de uma equipe especializada em atendimento a bebês prematuro sem uma UTIN, acessou-se, por meio da entrevista semiestruturada, a experiência materna, e, ao mesmo tempo, avaliou-se a experiência do serviço prestado pela equipe de cuidados. Já no estudo de Hagen et al. (2016), antes de serem aplicadas aos participantes, as entrevistas passaram por um estudo piloto (entrevista préteste), com o objetivo de verificar a eficácia das perguntas nelas contidas, corroborando, assim, o que refere Breakwell, Hammond, Fife-Scham e Smith (2010), que as entrevistas em pesquisa requerem uma abordagem muito sistemática da coleta, da análise e da descrição dos dados, o que possibilita ao entrevistador aumentar as chances de conseguir conclusões significativas, válidas e fidedignas. $\mathrm{O}$ pré-teste demonstrou que algumas perguntas da entrevista deveriam ser modificadas para se tornarem mais compreensíveis, sugerindo, também, o aumento do tempo da mesma, uma vez que o estudo investigou as diferenças e semelhanças no enfrentamento de mães e pais de crianças prematuras em uma unidade de terapia intensiva neonatal. Em todas as pesquisas da presente revisão, as entrevistas ocorreram na casa dos participantes ou nas UTINs (em um local privado), tendo apenas uma delas ocorrido por telefone, a pedido da mãe participante (Premji et al., 2017). Todas as entrevistas foram gravadas e posteriormente transcritas na íntegra. Já no estudo de Nelson e Bedford (2016), além de serem transcritas, o seu conteúdo foi enviado aos participantes, para que validassem a veracidade da transcrição para, em seguida, dar seguimento à análise das mesmas.

$\mathrm{Na}$ averiguação da categoria 10) "resultados", observa-se que, mesmo que os estudos discutam diferentes perspectivas sobre as experiências maternas em contexto de prematuridade, alguns resultados foram semelhantes. Diante disso, organizou-se a apresentação dos resultados em formato de subcategorias. Estas foram criadas por serem comuns aos diferentes estudos, e também por apresentarem os resultados mais significativos em termos de objetivo da presente revisão sistemática.

A subcategoria 10a) diz respeito aos "programas de atendimento especializado ao pré-termo", pois o estudo de Nelson e Bedford (2016) destaca a importância de se ter programas específicos de cuidado ao prematuro e à sua família, como, por exemplo, o NIDCAP. Os referidos autores indicam que este tipo de programa aumenta o conhecimento materno, dando-lhe confiança e a preparando para a alta. Já o estudo de Premji et al. (2017) confirma esta afirmação, mas de forma complementar, visto que, no mesmo, as mães não estavam preparadas para os cuidados de seus bebês em casa após a alta, pois a equipe de saúde não era especializada nos cuidados centrados na família e no RNPT, mas apenas a bebês a termo. Assim, nesses casos, houve falta de apoio da equipe com as mães dos RNP, pois a mesma não tinha os conhecimentos necessários para auxiliá-las, as quais, salienta-se, não tinham ciência do que era ter um bebê, nascido prematuramente.

Outro fator de impacto ao bom desenvolvimento da maternidade, nos casos de prematuridade, evidenciado no estudo de Nelson e Bedford (2016), é que, mesmo diante da possibilidade de fazerem parte dos programas de atenção e apoio ao prematuro, algumas mães não aceitaram participar. Segundo a pesquisa, elas deixaram de aderir aos mesmos por questões internas e externas a elas, não descritas no estudo. De outra parte, houve a ocorrência de não adesão às recomendações do programa por parte de alguns profissionais da equipe de saúde na realização das suas atividades laborais, o que ocasionou a quebra do cumprimento do programa, assim como do tipo de atendimento prestado às mães. 
A subcategoria 10b) foi dividida em "experiências durante a internação" e "experiências após a internação". A respeito das "experiências durante a internação", verificou-se, no estudo de Nelson e Bedford (2016), que, apesar das mães participantes do estudo terem a possibilidade de participar do programa NIDCAP, o qual capacita profissionais a atuarem de forma especializada no cuidado e atenção ao RNPT e à sua família, além de proporcionar um espaço de inserção dos pais na UTIN, em tempo integral, para realização dos cuidados de seus bebês, algumas delas relataram que passar muitas horas no hospital fez com que tivessem dificuldades de gerenciar situações externas ao contexto hospitalar, como, por exemplo, dinâmica familiar (cuidado de outros filhos que estavam em casa) e conjugal, quando o parceiro não estava inserido no contexto de cuidado do RNPT hospitalizado.

Já no estudo de Hagen et al. (2016), as dificuldades encontradas foram que as mães não conseguiam falar sobre os cuidados infantis, tinham a sensação de alienação e problemas de ligação com o bebê.Também se percebeu que mães que tinham tido partos complicados anteriores apresentaram mais dificuldades no cuidado do prematuro, em comparação com aquelas que tiveram bebês a termo. O pós-parto também foi citado como uma dificuldade, sendo denominado como estressante, nos casos em que os pais não estavam presentes. No estudo de Finlayson et al. (2014), as mães tiveram que lutar para encontrar seu "lugar" dentro da configuração da UTIN, e a chegada inesperada do bebê e o ambiente hospitalar causaram dificuldades em desempenhar o papel materno, resultando em uma sensação de desamparo. As mães sentiram-se vulneráveis e expostas a lutas de poder e/ou relacionamentos silenciosos para com a equipe de enfermagem; sentiam-se sensíveis na entrega do cuidado e na troca de informações, e, na busca de manter o equilíbrio e proteger o bebê, estavam em permanente estado de ansiedade.

Já na subcategoria "experiências após a internação", Premji et al. (2017) observaram que as mães relataram o quão difícil foi prestar os cuidados aos bebês nascidos pré-termo em casa, após a alta, principalmente no que se refere à alimentação. $\mathrm{O}$ seu estudo foi realizado, contudo, com uma equipe de saúde que não era especializada em atendimento a RNPT. Para Spinelli et al. (2016), uma dificuldade enfrentada pelas mães foi o fato de considerar que a sua maternidade estava atravessada pelo "Outro Institucional". O hospital é a "grande mãe" especializada, que rege os comportamentos maternos, e isso pode afetar a construção da identidade materna, a imagem da mãe para com o bebê, e o desenvolvimento do vínculo entre a díade, impedindo a transição para a parentalidade, ameaçando, assim, a reorganização da identidade materna, conforme propõe a teoria da Constelação da Maternidade (Stern, 1997). A dependência do "Outro Institucional" pode levar as mães de volta a um estado infantil, por sentirem que precisam da permissão da instituição para desempenhar um papel na vida do bebê.

Já na categoria 10c), sobre as "experiências positivas no contexto de prematuridade", o estudo de Guillaume et al. (2013) revelou que as mães sentiram que houve um encontro solidário entre pais e equipe de cuidadores, possibilitando o desenvolvimento de um bom vínculo com o bebê. No nascimento prematuro, as primeiras semanas são fundamentais, pois o vínculo da díade se inicia por meio da equipe de cuidados. A pesquisa revelou que as mães sentiram que os enfermeiros prestaram cuidados não apenas ao bebê, como também aos familiares, assim como desenvolveram um canal de comunicação eficaz que auxiliou na diminuição do estresse e permitiu a interação com o recém-nascido, além de perceberem que o cuidado prestado era regular e adaptado, dado este também encontrado no estudo de Premji et al. (2017). Esses achados remetem ao conceito de Winnicott (2006), sobre a mãe suficientemente boa: a equipe foi suficiente nos cuidados, não era negligente e nem excessiva, era adequada para com as necessidades das mães. Outro aspecto positivo encontrado no trabalho de 
Hagen et al. (2016) é que as mães relataram que o enfrentamento do contexto de prematuridade e hospitalização era menos doloroso quando o pai estava presente no ambiente hospitalar. No estudo de Nelson e Bedford (2016), as mães ressaltaram a importância de terem tido um espaço privado em uma sala individual, na UTIN, para ficarem mais à vontade com seus bebês, relatando que, neste momento, sentiam que seus filhos eram seus e não da equipe de saúde, como geralmente ocorria.

$\mathrm{Na}$ categoria 10d), "papel da equipe / instituição", nota-se que o papel institucional, representado pela equipe de cuidados, é fundamental nos casos de prematuridade. Para que ocorra o desenvolvimento "saudável" do vínculo e da interação entre a díade mãe-bebê nesses casos, Nelson e Bedford (2016) referem que a equipe deve ser sensível às necessidades maternas, pois são eles que constroem a ponte de ligação entre a mãe e o recém-nascido. As participantes relataram que foram bem cuidadas pela equipe de saúde e que, por isso, sentiram-se fazendo parte da mesma, como se fossem uma família. De acordo com a pesquisa de Spinelli et al. (2016), as mães precisam ser habilitadas e empoderadas a desenvolverem uma identidade materna para que os demais temas da Constelação da Maternidade também possam acontecer de forma natural. Já no estudo de Premji et al. (2017), a equipe de enfermagem não era especializada em RNPT, incidindo negativamente na forma de conduzir o processo de forma constante. Além disso, havia uma significativa rotatividade de trabalhadores no setor, o que fazia com que as mães não tivessem um profissional de referência, e cada um deles orientava as mães de forma diferente, deixando-as estressadas, ansiosas, confusas e inseguras com relação aos cuidados do bebê.

No estudo de Nelson e Bedford (2016) não era a falta de capacitação que impedia os profissionais de atuarem de forma especializada no atendimento ao RNPT e à sua família, mas, sim, a não adesão ao programa de capacitação e cuidados oferecido pelo hospital. Havia o programa, mas nem todos os profissionais aderiram, na rotina hospitalar diária, às práticas indicadas pelo protocolo. As mães relataram que isso impactou negativamente nos cuidados da equipe para com os bebês, o que incidiu, consequentemente, no desenvolvimento do vínculo da díade. Por fim, o estudo de Premji et al. (2017) propõe, ao final de seu trabalho, que os protocolos de atendimento ao prematuro e à sua família na UTIN são essenciais ao bom acolhimento e à recuperação para a alta, mas que também se faz necessário um protocolo de orientação no que tange aos principais cuidados prestados ao bebê no momento da saída do hospital, a serem aplicados em casa. Para os autores, a instituição tem o poder de auxiliar e amenizar essa situação, pois, nesse momento, as mães têm apresentado muitas dificuldades.

\section{Considerações Finais}

A partir dos dados, verificou-se a escassez de estudos enfatizando as "experiências maternas" em contexto de prematuridade. Estas, apareceram de forma secundária nas publicações. Por outro lado, os estudos têm demonstrado uma preocupação crescente com a qualidade do serviço e com a capacitação da equipe de cuidados. As pesquisas em diferentes continentes e países, têm se preocupado com os altos índices de nascimento de RNPT, e, consequentemente, com a forma mais adequada de se realizar o tratamento destes pacientes, que, na maioria dos casos, ficam internados em uma UTIN.

Entretanto, notou-se que, além da tecnologia e de uma boa estrutura física, como, por exemplo, leitos individualizados para a família ter privacidade com o bebê, um fator impactante descrito em todos os artigos foi que as pessoas que compõem a equipe de cuidados no ambiente de UTIN devem ter uma disponibilidade emocional para com os envolvidos. A atitude subjetiva de cada membro das equipes acaba por afetar os resultados positivos do trabalho em grupo. Esses aspectos levam à reflexão do quão fundamental é a presença do psicólogo no ambiente de UTIN nos casos de prematuridade, pois este profissional tem condições de realizar 
um trabalho de sensibilização para que a equipe se conscientize do papel no desenvolvimento da relação inicial entre a mãe e seu bebê, e do quanto isso impacta no bom desenvolvimento emocional da criança. Entretanto, tendo em vista que somente uma pesquisa foi realizada por psicólogos na presente revisão, sugere-se que outros estudos sejam também produzidos e publicados por profissionais desta área. Neste sentido, tendo em vista que a maioria dos artigos que compuseram essa pesquisa foram escritos por profissionais da área da medicina, infere-se que há a necessidade de uma maior participação do psicólogo no ambiente hospitalar, além de uma maior divulgação dos trabalhos realizados por estes profissionais.

É possível que o número de pesquisas pudesse ter sido ainda maior, uma vez que a exclusão de artigos que não tratavam de pesquisas empíricas pode ter sido uma limitação deste trabalho, pois se deixou de analisar estudos de caso e relatos de experiências, modos característicos de estudar o fenômeno da psicologia. Outro dado interessante é que houve predomínio total dos artigos escritos em inglês, confirmando ser este o idioma universal em termos de publicação. Entretanto, esta pode ser outra limitação do presente estudo, pois optou-se por buscar somente estudos em inglês. Talvez se tivessem sido usados descritores em português, pudessem ter sido encontradas pesquisas brasileiras sobre o tema. Contudo, como praticamente todos os estudos são publicados com o título e o resumo em inglês, acreditou-se que não teria uma discrepância tão grande com o atual trabalho. Sugere-se, entretanto, este tipo de pesquisa para outros pesquisadores. Verificou-se, também, a escassez de artigos relacionados ao tema proposto, o que, contudo, pode ser outra limitação do estudo, pois a pesquisa ocupou-se dos artigos empíricos, publicados apenas nos últimos cinco anos. Se esta tivesse abarcado os manuais técnicos do SUS e pesquisas nos últimos dez anos, certamente teríamos um cenário diferente do aqui apresentado.

Conclui-se que existe um desejo por parte dos profissionais da saúde (enfermeiros, médicos, psicólogos) em pesquisar a presente temática, e dentro do recorte dessa pesquisaque abrangeu 6 artigos para discussão- há poucas pesquisas por parte dos trabalhadores da psicologia. No entanto, há alguns anos o governo brasileiro vem desenvolvendo práticas interventivas mais humanizadas, dentre elas destacam-se: Manual Técnico da Atenção Humanizada ao Recém-Nascido de baixo peso (Método Canguru) (Ministério da Saúde, 2014), a Rede Cegonha (Ministério da Saúde, 2011b) e o programa de Estratégia QualiNEO (Portal da Saúde, 2017), com participação ativa dos profissionais da psicologia como parte da equipe assistencial à mãe e familiares dos RNPT.

\section{Referências}

Almeida, T. S. O., Lins, R. P., Camelo, A. L., \& Mello, D. C. C. L. (2013). Investigação sobre os fatores de risco da prematuridade: Uma revisão sistemática. Revista Brasileira de Ciências da Saúde, 17(3), 301-308. doi:10.4034/RBCS.2013.17.03.14

Bowlby, J. (1997). Formação e rompimento dos laços afetivos (3a ed.). São Paulo: Martins Fontes

Brazelton, T. B., \& Cramer, B. (1992). As primeiras relações. São Paulo, SP: Martins Fontes.
Breakwell, G. M., Hammond, S., Fife-Scham, C., \& Smith, J. A. (2010). Métodos de pesquisa em psicologia. Porto Alegre, RS: Artmed.

Fernandes, R. T., Lamy, Z. C., Morsch, D., Lamy Filho, F., \& Coelho, L. F. (2011). Tecendo as teias do abandono: Além das percepções das mães de bebês prematuros. Ciência \& Saúde Coletiva, 16(10), 40334042. doi:10.1590/s141381232011001100008

Ferrari, A. G., \& Donelli, T. M. S. (2010). Tornar-se mãe e prematuridade: 
Considerações sobre a constituição da maternidade no contexto do nascimento de um bebê com muito baixo peso. Contextos Clínicos, 3(2), 106-112.

doi:10.4013/ctc.2010.32.04

Finlayson, K., Dixon, A., Smith, C., Dykes, F., \& Flacking, R. (2014). Mothers' perceptions of family centred care in neonatal intensive care units. Sexual \& Reproductive Healthcare, 5(3), 119-124. doi:10.1016/j.srhc.2014.06.003

González-Serrano, F., Lasa, A., Hernanz, M., Tapia, X., Torres, M., Castro, C., \& Ibañez, B. (2012). Maternal attachment representations and the development of very low birth weight premature infants at two years of age. Infant Mental Health Journal, 33(5), 477-488. doi:10.1002/imhj.21345

Guillaume, S., Michelin, N., Amrani, E., Benier, B., Durrmeyer, X., Lescure, S., ... Zana-Taïeb, E. (2013). Parents' expectations of staff in the early bonding process with their premature babies in the intensive care setting: A qualitative multicenter study with 60 parents. $B M C$ Pediatrics, 13(1), 18-26. doi:10.1186/14712431-13-18

Hagen, I. H., Iversen, V. C., \& Svindseth, M. F. (2016). Differences and similarities between mothers and fathers of premature children: A qualitative study of parents' coping experiences in a neonatal intensive care unit. BMC Pediatrics, 16(1), 92-100. doi:10.1186/s12887-016-0631-9

Lebovici, S. (1987). A constituição do elo entre a mãe e o recém-nascido. In $\mathrm{S}$. Lebovici (Org.), O bebê, a mãe e o psicanalista (pp. 115-126). Porto Alegre, RS: Artes Médicas.

Ministério da Saúde. Secretaria de Atenção à Saúde. Departamento de Ações Programáticas Estratégicas. (2014). Atenção à Saúde do Recém-Nascido: Guia para os Profissionais de Saúdel Ministério da Saúde. Secretaria de Atenção à Saúde. Departamento de Ações Programáticas Estratégicas. Brasília: Editora do Ministério da Saúde.
Ministério da Saúde. (2011a). Atenção humanizada ao recém-nascido de baixo peso: Método Canguru. 2a. ed. Brasília, DF: Editora do Ministério da Saúde.

Ministério da Saúde. Gabinete do Ministro. (2011b) PORTARIA № 1.459, DE 24 DE JUNHO DE 2011: Instituto no âmbito do Sistema Único de Saúde - SUS - a Rede Cegonha. Recuperado de http://bvsms.saude.gov.br/bvs/saudelegis/g m/2011/prt1459_24_06_2011.html

Moher, D., Liberati, A., Tetzlaff, J., Altman, D. G., The PRISMA Group. (2009). Preferred reporting items for systematic reviews and meta-analyses: The PRISMA statement. Annals of Internal Medicine, 151(4), 264-269. doi:10.7326/0003-4819151-4-200908180-00135

Moreira, M. E. L., Lopes, J. M. A., \& Carvalho, M. (2004). O recém-nascido de alto risco: Teoria e prática do cuidar. Rio de Janeiro, RJ: Editora Fiocruz.

Nelson, A. M., \& Bedford, P. J. (2016). Mothering a preterm infant receiving NIDCAP care in a level III newborn intensive care unit. Journal of Pediatric Nursing, 31(4), 271-282. doi:10.1016/j.pedn.2016.01.001

Portal da Saúde. (2017). Ministério da Saúde Lança Estratégia para Reduzir Mortalidade Neonatal. Recuperado de http://portalsaude.saude.gov.br/index.php/o -ministerio/principal/secretarias/sas/sasnoticias/29023-ministerio-da-saude-lancaestrategia-para-reduzir-mortalidadeneonatal.

Premji, S. S., Currie, G., Reilly, S., Dosani, A., Oliver, L. M., Lodha, A. K., \& Young, M. (2017). A qualitative study: Mothers of late preterm infants relate their experiences of community-based care. PLOS ONE, 12(3), e0174419-e0174419. doi: 10.1371/journal.pone.0174419

Silveira, D. T., \& Córdova, F. P. (2009). A pesquisa científica. In T. E. Gerhardt, \& D. T. Silveira (Orgs.), Métodos de pesquisa (pp. 31-42). Porto Alegre, RS: Editora da UFRGS.

Soulé, M. (1987). O filho da cabeça, o filho imaginário. In T. Brazelton, B. Cramer, L. 
Kreisler, R. Shappi, \& M. Soulé (Orgs.), A dinâmica do bebê (pp. 132-170). Porto Alegre, RS: Artes Médicas.

Spinelli, M., Frigerio, A., Montali, L., Fasolo, M., Spada, M. S., \& Mangili, G. (2016). 'I still have difficulties feeling like a mother': The transition to motherhood of preterm infants mothers. Psychology \& Health, 31(2), 184-204. doi:10.1080/08870446.2015.1088015

Stern, D. N. (1997). A constelação da maternidade: O panorama da psicoterapia pais/bebê. Porto Alegre, RS: Artes Médicas.
Unicef Brasil. Fundo das Nações Unidas para a Infância. (2013). Prematuridade e suas possíveis causas. Recuperado de https://www.unicef.org/brazil/pt/activities 27450.html

Wanderley, D. B. (1997). Palavras em torno do berço: Intervenções precoces bebêe família. Salvador, BA: Algama.

Winnicott, D. (2006). Os bebês e suas mães (3a.ed.). São Paulo: Martins Fontes.

Word Health Organization. [WHO]. (2018). Nacimientos prematuros. Recuperado de http://www.who.int/es/news-room/factsheets/detail/preterm-birth

\section{Dados sobre as autoras:}

- Denice Bortolin: Doutorado em Psicologia Clínica, pela Universidade do Vale do Rio dos SinosUnisinos. Docente no curso de Psicologia pela Faculdade Meridional- IMED.

- Tagma Marina Schneider Donelli: Doutorado em Psicologia pela Universidade Federal do Rio Grande do Sul. Docente na Universidade do Vale do Rio dos Sinos-Unisinos

- Carine Tabaczinski: Pós-graduanda em Psicologia e Maternidade pela UNIARA, bacharel em psicologia pela Faculdade Meridional- IMED. 